\title{
Reinstating study context produces unconscious influences of memory
}

\author{
SCOTT W. ALLEN and LARRY L. JACOBY \\ McMaster University, Hamilton, Ontario, Canada
}

\begin{abstract}
Having read a word does more to benefit its later perceptual identification when many, rather than few, of the words in the test list have been previously read. Some have suggested that this proportion overlap effect is produced by an intentional use of recognition memory or recall in the perceptual identification task. Contrary to this account, we found that words that are easily recognized (words generated from an anagram at study) do not gain more from increasing overlap than do words that are poorly recognized (words read at study). These findings are problematic for claims that word perception relies on a module, such as a logogen system, that is separate from the rest of memory.
\end{abstract}

Evidence of memory is often shown by performance on an indirect test, although a direct test reveals no evidence of memory (for reviews, see Johnson \& Hasher, 1987; Richardson-Klavehn \& Bjork, 1988). For example, reading a word makes it more likely that a person will be able to identify the word later when it is flashed for a test of perceptual identification, and that effect on perception is observed even when the person does not recognize the word as previously read (e.g., Jacoby \& Dallas, 1981). The test of perceptual identification is an indirect test of memory because the instructions refer only to the task at hand and do not refer back to a particular prior event, although the subject's performance on the task might be influenced by memory for that prior event. Recognition and recall are direct tests of memory because the instructions refer to a target event in the personal history of the subject and ask the subject to consciously recollect that earlier event. We use the phrase unconscious influences of memory (e.g., Jacoby \& Kelley, 1987) to refer to effects of a prior experience that arise on an indirect test of memory although a person is unable to recall or recognize the relevant prior experience. Others (e.g., Graf $\&$ Schacter, 1985) have referred to such effects as effects on implicit memory.

A major problem for using indirect tests as a means of investigating unconscious influences of memory is that performance on these tests might sometimes reflect aware influences of memory that are undetected by the experimenter. Although not instructed to use memory for a particular prior event, subjects given an indirect test of memory might sometimes discover the relation between

This research was supported by the Natural Sciences and Engineering Research Council of Canada through a postgraduate scholarship to the first author and an operating grant to the second author. We thank H. L. Roediger III and A. Richardson-Klavehn for their comments on an earlier draft of this article. Requests for reprints may be sent to Larry L. Jacoby, Department of Psychology, McMaster University, Hamilton, Ontario L8S 4K1, Canada. study and test and intentionally use memory much as they would for a direct test of memory. The problem is similar to the one that has plagued attempts to show unconscious perception. Indeed, because of the possibility of awareness undetected by the experimenter, Holender (1986) argues that there is no convincing evidence for the existence of unconscious perception. Similarly, in studies of memory, Richardson-Klavehn and Bjork (1988) note that many effects ascribed to unconscious influences of memory might be contaminated by conscious recollection. For example, the enhanced perceptual identification performance of old, relative to new, words might sometimes reflect the quite deliberate and conscious retrieval of studied words.

Finding factors that produce dissociations between performance on indirect and direct tests of memory allows one to circumvent some of the problems produced by the possibility of undetected awareness. For example, there is evidence that manipulations of the processing of an item during its prior presentation can have effects on the performance of an indirect test that are the opposite of those on a direct test of memory. Reading a word does more to benefit its later identification than does generating the word as an antonym of a presented word, but it produces poorer recognition memory performance (Jacoby, 1983b). Similarly, Roediger and his colleagues (e.g., Roediger $\&$ Blaxton, 1987) have observed dissociations between effects of prior processing on fragment-completion and recognition memory performance that can be described as reflecting the compatibility of study and test processing. Dissociations of this sort provide evidence for the existence of unconscious influences of memory. However, the use of dissociations is limited to showing differences between factors influencing performance on indirect and direct tests of memory. The possibility of undetected awareness remains as a problem for interpreting effects on an indirect test that are in the same direction as are effects on a direct test of memory. The problem is an important one, because similarities between effects can be 
as useful as differences are for choosing between theoretical accounts of the relation between performance on the two types of test.

In this paper, we consider the effects on identification performance of reinstating study context at the time of test and show that those effects are not always mediated by an intentional use of memory. The presence of context effects on an indirect test of memory is of theoretical importance. A popular account of dissociations between effects on indirect and direct tests of memory is to claim that performance on the two types of test relies on different memory representations or different memory systems. Conscious recollection of a prior event, as measured by recognition memory or recall performance, is said to rely on episodic memory, whereas word identification or word-fragment-completion performance (indirect tests of memory) is said to rely on procedural or semantic memory (e.g., Cohen \& Squire, 1980; Tulving, 1983). Procedural and semantic memory summarize earlier experience in the form of general, abstract representations that do not preserve the details of any particular prior encounter with an item. Details of that sort are preserved only by the more specific memory representations that comprise episodic memory. Consequently, reinstating study context at the time of test should not influence performance on an indirect test of memory.

Rather than proposing separate memory representations, others (e.g., Jacoby, 1983a, 1983b; Jacoby \& Brooks, 1984; Whittlesea, 1987) claim that performance on perceptual tasks, as well as recall and recognition memory performance, can rely on memory for prior episodes. This episodic view of perception might seem incompatible with the existence of dissociations between effects on perceptual tasks and recognition memory performance. However, by referring to episodic effects on perception, it is not necessary to imply that those effects depend on a person's awareness of memory for a prior episode as measured by a test of recognition memory. When proposing an episodic view of perception, the term episodic is used only to indicate that effects are specific to memory for a particular prior episode rather than being more general effects that arise from the priming or activation of some abstract representation, such as a logogen or schema. An episodic view of perception treats the issue of the specificity of effects as separate from that of a subject's awareness of the source of those effects (e.g., Jacoby \& Kelley, 1987). By an episodic view, reinstating study context at the time of test should be important for retrieval of memory for prior episodes and, consequently, should influence performance on an indirect test of memory.

Effects of reinstating study context at the time of test on recognition memory and recall performance are commonly found and have been interpreted as showing the importance of test cues for retrieval of memory for prior episodes (e.g., Tulving, 1983). Similar effects have also been found with amnesics and normals using indirect measures of memory, such as reading times for words and sentences (Moscovitch, Winocur, \& McLachlan, 1986) and stem-completion performance (Graf \& Schacter, 1985). Results from those experiments have been used as evidence that amnesics are capable of learning new associations. Jacoby (1983a) found an effect of manipulating test context on perceptual identification performance. He varied the proportion of words presented for a test of perceptual identification that had been earlier read and found that earlier reading of a word caused a larger benefit on its later identification when nearly all, rather than very few, of the test words were from the earlier read list. This effect of "proportion overlap" between the study and test lists was taken as showing that reinstating study context is important for perceptual identification performance and was used to support the argument that, like recognition memory and recall, effects of prior experience on perceptual identification performance reflect memory for prior episodes.

However, interpretation of the effect of reinstating study context on performance of indirect tests of memory is controversial. The effect on indirect tests is in the same direction as that on direct tests and, so, may be mediated by an intentional use of memory. Evidence of new learning revealed by indirect tests of memory (e.g., Graf \& Schacter, 1985) might result from some subjects' intentional use of memory. Consistent with this possibility, Schacter and Graf (1986) suggest that effects of reinstating study context occur for moderate, but not severe, amnesics. Some have argued that the proportion overlap effect in perceptual identification performance occurs not because of an effect of reinstating study context on the use of an episodic basis for word identification itself but, rather, because of a contamination of performance on the perceptual identification task by an intentional use of recognition memory or recall (e.g., Schacter, 1985). For example, awareness of the relation between study and test might result in the use of list recognition as a criterion for responding on the test of perceptual identification. When subjects are aware that most test words were earlier read, they might be more willing to output a word as a perceptual identification response if they recognize the word as one that was earlier read. This reliance on list recognition would produce effects of episodic memory on perceptual identification performance that are not truly perceptual effects. Such effects could also result when subjects identify only a few letters from a word flashed for a test of its perceptual identification and then use those letters as a cue to intentionally recall an earlier read word. These intentional uses of memory to aid perceptual identification performance might be made more likely by increasing proportion overlap between study and test; subjects might be more likely to catch on to the relation between study and test with a higher proportion overlap in the words used for the two. By this intentional memory interpretation, an aware use of memory adds to the effects of priming to determine overall performance.

The experiments reported in this article were designed to discriminate between the alternative accounts of the ef- 
fects of proportion overlap on word identification. To do this, we took advantage of the finding that some manipulations have an effect on perceptual identification performance that is opposite to the effect that they have on recognition memory and recall performance (e.g., Jacoby, 1983b). In particular, words were presented either in their normal form to be read or as anagrams to be solved. We expected that reading a word would later produce better perceptual identification performance but poorer recognition memory performance than would generating a word as a solution for an anagram. Crossing the type of processing done at study (reading vs. generation from an anagram) with the context at test (high- vs. low-proportion overlap) allows for differential predictions from the two explanations of proportion overlap effects.

By the intentional memory interpretation, words generated from an anagram will benefit more in later identification performance from high-proportion overlap than will words that were read when earlier presented. This prediction is made because words generated at study are known to be better recognized or recalled than are words that are read; it is an increased reliance on these intentional uses of memory that is said to be responsible for the effects on identification of increasing proportion overlap. In contrast, the episodic view holds that the critical factor influenced by the manipulation of proportion overlap is the similarity of study and test context. Increasing the similarity of those contexts by increasing proportion overlap is said to make it more likely that the perceptual identification of a word will be enhanced by retrieval of memory for its earlier processing. The episodic view predicts that words that were generated will not benefit any more from increasing proportion overlap than will words that were read.

\section{EXPERIMENT 1}

\section{Method}

Subjects and Design. The subjects were 48 students enrolled in an introductory cognition course who participated in the experiment for course credit. Twenty-four subjects were randomly assigned to each of two (high and low overlap) test conditions. In the high-overlap condition, $90 \%$ of the words presented for a test of perceptual identification had been presented in an earlier list, whereas, in the low-overlap condition, only $20 \%$ of the perceptual identification test words had been presented in an earlier list. The manipulation of test overlap was factorially combined with a withinsubject manipulation of the earlier presentation of words (old-read, old-generated, and new) that were later presented for the test of perceptual identification. To avoid ceiling effects, we replaced 6 subjects ( 4 from the high-overlap group and 2 from the low-overlap group) whose performance on the new items in the perceptual identification test was better than $70 \%$ correct. The necessity to replace the subjects resulted from a failure on the part of the experimenter to set the presentation duration of the test words at a level that was low enough for those subjects. Replacing the subjects did not change the pattern of the results.

There were four phases in Experiment 1. In Phase 1, a test of perceptual identification was given so as to specify, for each subject, a presentation duration to be used for a later test of perceptual identification. A long list that included words that were to be read and anagrams that were to be solved was presented in Phase 2. In Phase 3, a test of perceptual identification was given that included words that were read, words that were generated as a solution to an anagram in Phase 2, and new words. The locus of the manipulation of proportion overlap was in this third phase of the experiment. Either $90 \%$ or $20 \%$ of the words presented for the test of perceptual identification in Phase 3 were old. A test of list recognition was given in Phase 4.

Materials. A pool of 194 five-letter nouns that occur infrequently in written natural language (1-5 occurrences per million words), as scaled by Thorndike and Lorge (1944), was used as materials. Twenty-four words from that pool were used in the perceptual identification test given in Phase 1. Of the remaining 170 words, 90 words were used to construct a list that was to be presented in Phase 2. Half of the words on that list were presented in their normal form to be read, and half were presented as anagrams to be solved. To construct anagrams, words were presented with the second and fourth letters in their proper positions and underlined. The remaining letters in a word presented as an anagram were randomly rearranged. Constraining the order of letters in an anagram had two purposes: it made the anagrams somewhat easier to solve, and, more importantly, it allowed anagrams to be constructed such that each anagram had only one correct solution.

For purposes of later phases of the experiment, the list presented in Phase 2 can be treated as containing 20 critical words (10 read and 10 generated) and 70 filler words (35 read and 35 generated). Those 20 critical words, along with 10 critical new words (words not presented earlier), were always included in a list of 100 words presented for the test of perceptual identification given in Phase 3. Other than the critical words, the choice of words to be included in that test was determined by the manipulation of proportion overlap. For high-proportion overlap, the test list included the 70 filler words ( 35 read and 35 generated) that were presented in Phase 2 , making a total of 90 old words and 10 new words. For lowproportion overlap, the test list included 70 new words (not seen before in the experiment), making a total of 20 old words and 80 new words. Again, critical words were those that appeared, across subjects, in both the high- and the low-overlap test lists. It was perceptual identification of those critical words that was of primary interest.

Multiple list formats were used so as to avoid confounding words with experimental conditions. Three formats for a list presented in Phase 2 were constructed by using a Latin square design, such that sets of 10 critical words were rotated through the conditions (defined in reference to Phase 3 ) of old-read, old-generated, and new. Two additional formats were constructed by interchanging the 70 words that served as old filler words in the high-overlap test list with the 70 words that served as new words in the low-overlap test list. The factorial combination of these two types of format produced six different formats for the list presented in Phase 2. Also, two different random orders of words presented for the test of perceptual identification given in Phase 3 were used. Each combination of list format and test order was used equally often.

The test of list recognition memory that was given in Phase 4 included the 90 words that had been presented in Phase 2 (45 read and 45 generated), along with 90 new low-frequency words. Old and new words were randomly intermixed and typed on a sheet of paper.

Procedure. An Apple computer interfaced with a Zenith monitor was used to present stimuli. Character size was approximately $5.7 \times 6.6 \mathrm{~mm}$; words were presented in lowercase letters. The subjects were seated such that their viewing distance was 70 to $75 \mathrm{~cm}$. The use of a monitor makes stimulus presentation reliant on raster scan technology and limits the presentation of stimuli to a multiple of $1 / 60 \mathrm{sec}$. This means that the presentation durations that will be stated are only approximate. 
In Phase 1, a perceptual identification test was used to allow the experimenter to set, for each subject, a target duration that would allow approximately $50 \%$ correct identification of test words. For that test, the subjects initiated the presentation of a test word by pressing the space bar on the computer keyboard. Pressing the space bar resulted in a 750-msec presentation of a set of fixation bars (-- ---) that surrounded the location at which the test word would be presented. The test word was then presented in that location and followed by a rectangle of randomly placed dots large enough to cover the entire space that had been occupied by the test word. The visual mask remained on the screen for 500 msec. After attempting to report the test word, the subject pressed the space bar to initiate the next presentation. Each of the first eight test words was presented for a duration of $50 \mathrm{msec}$. This duration was then adjusted up or down for the second set of eight test words and again for the third set of eight test words in an attempt to find a presentation duration that would produce approximately $50 \%$ accuracy.

The subjects were told that we were interested in the relation between their ability to identify words that were briefly flashed and their ability to solve anagrams. The list presented in Phase 2 included words that were to be read and anagrams that were to be solved. The subjects were misinformed that the time they required to read words was to be used as a baseline against which to compare the amount of time they required to solve anagrams. Times were not actually recorded. The presentation of items was again controlled by the subject's pressing the space bar. When the subject pressed the space bar, a word or an anagram appeared on the screen and remained there until he/she pressed the space bar to indicate that the experimenter had said "correct" to the subject's reading of a word or solving of an anagram. The subject then pressed the space bar again to initiate presentation of the next item. The subject was told that the second and fourth letters of the anagrams would always be underlined and that the underlined letters were in their proper positions. The task was to rearrange the remaining three letters until the subject could report the solution word for the anagram. If the subject had difficulty solving an anagram, the experimenter gave the first letter of the solution word as a hint. On the rare occasions when the subject could still not solve the anagram, a semantic hint was then given (e.g., "a kind of hat" for "beret"). Given these hints, failure to solve an anagram was quite rare (less than $1 \%$ ) and generally occurred only when a subject was completely unfamiliar with a solution word. For words presented in normal form, the subject was told to read the words aloud.

The procedure for the test of perceptual identification given in Phase 3 was generally the same as for the test given in Phase 1 . The presentation duration of test words was set at a value chosen from the earlier phase to produce approximately $50 \%$ identification accuracy for new test words. The subjects in the high-overlap condition were told that nearly all of the words that were to be presented for the test of perceptual identification were words that they had read or words that they had given as a solution to an anagram in the earlier presented list. The subjects in the low-overlap condition were not told that the test of perceptual identification would include any words from the earlier presented list.

In the final phase of Experiment 1, the test of recognition memory was given. Words were presented on a sheet of paper. The subjects were told to circle all the words that they had earlier read or had earlier given as a solution to an anagram during the experiment.

The significance level for all tests was set at $p<.05$.

\section{Results and Discussion}

Recognition memory. The recognition memory results are shown in Table 1. Although all subjects were given the perceptual identification test, recognition memory data were collected from only 23 of the subjects in the highoverlap condition due to experimenter error.
Table 1

Percent Correct Recognition Memory and

Perceptual Identification Performance in Experiment 1

\begin{tabular}{cccc}
\hline \multirow{2}{*}{$\begin{array}{c}\text { Overlap } \\
\text { Condition }\end{array}$} & \multicolumn{3}{c}{ Item Type } \\
\cline { 2 - 3 } New & Old-Read & Old-Generated \\
High & 93.2 & 77.4 & 85.0 \\
Low & 91.3 & 55.9 & 74.5 \\
\multicolumn{5}{c}{ Recognition Memory } \\
High & Perceptual Identification \\
Low & 45.0 & 80.0 & 70.0 \\
\hline
\end{tabular}

For the high-overlap condition, words that were old on the test of recognition memory had been read or given as a solution to an anagram in Phase 2 and had also been presented for the test of perceptual identification in Phase 3. The same holds true for the critical items in the low-overlap condition. The second presentation of those words for the test of their identification is likely to diminish the difference in recognition memory between words that were earlier read and those that were generated as the solution to an anagram. To produce the most accurate estimate of the difference in recognizability between read and generated words at the time of the perceptual identification test (when there had been only one prior presentation), only the noncritical items from the low-overlap condition were included in the analysis. ${ }^{1}$ An analysis of recognition performance for those words showed that words that were earlier generated as a solution to an anagram were much more likely to be called "old" $(70.9 \%)$ than were words that were earlier read $(50.0 \%)[t(23)=$ 6.58]. (Note that these means do not include the critical items that were seen in Phase 2, whereas the means in Table $1 \mathrm{do}$.) The probability of calling a new word "old" was $8.8 \%$. The advantage in recognition memory performance for words that had been generated as a solution to an anagram over those that were read is consistent with the results of other experiments (e.g., Jacoby, 1978, 1983b; Slamecka \& Graf, 1978).

Perceptual identification. The presentation duration employed for perceptual identification ranged from 17 to $50 \mathrm{msec}$ and had a mean of $25.5 \mathrm{msec}$. The perceptual identification results are shown in Table 1. A first analysis of those data included only words that were earlier read and words that were new on the test of perceptual identification, conditions included in the experiment reported by Jacoby (1983a). The analysis of those results revealed the same pattern of effects as that reported by Jacoby, although the effects in our experiment were somewhat weaker than those in his. Words that had earlier been read were more likely to be correctly identified $(75.8 \%)$ than were words that were new $(46.5 \%)$ on the test of perceptual identification $\left[F(1,46)=106.1, M S_{\mathrm{c}}=195.2\right]$. The main effect of proportion overlap between study and test did not approach significance $(F<1)$. More importantly, there was a marginally significant interaction be- 
tween prior presentation and proportion overlap $[F(1,46)$ $\left.=3.89, M S_{\mathrm{e}}=195.2, p<.10\right]$. Earlier reading of a word did somewhat more to enhance its later identification when the majority of the words tested had been earlier presented. A one-tailed $t$ test revealed that perceptual identification of words that were earlier read was higher in the high-overlap condition than in the low-overlap condition $[t(46)=1.78]$. The use of a one-tailed test is justified because the direction of the difference was predicted.

Turning to the two types of old words, the effect of reading versus generating a word on perceptual identification was the opposite of that on recognition memory performance. An analysis of the effects on perceptual identification showed that read words were significantly more likely to be identified $(75.8 \%)$ than were generated words $(66.3 \%)\left[F(1,46)=15.6, M S_{c}=141.1\right]$ and that the high-overlap group performed marginally better $(75 \%)$ than did the low-overlap group $(67.1 \%)[F(1,46)=2.95$, $M S_{\mathrm{e}}=510.7, p<.10$; if tested by a one-tailed $t$ test, this difference would be significant at $p<.05$ ]. The interaction between earlier reading or earlier generating a word and proportion overlap did not approach significance $(F<1)$.

The intentional memory interpretation of the proportion overlap effect cannot explain effects on perceptual identification that were observed in this experiment. By the intentional memory interpretation, words that were generated should benefit more from high-proportion overlap than do words that were earlier read. This is because intentional uses of memory (such as the use of recognition memory as a criterion for responding) are said to be responsible for the effect of increasing proportion overlap. However, the effect of increasing proportion overlap was no larger for words that were earlier generated (words that were likely to be recognized) than it was for words that were earlier read (words that were unlikely to be recognized).

A difficulty for choosing between accounts of the proportion overlap effect is that the effect observed in Experiment 1 was a rather weak one. Experiment 2 was designed to remove this difficulty by producing a more reliable effect of proportion overlap.

\section{EXPERIMENT 2}

A strong manipulation of proportion overlap might require that subjects be able to perceptually identify most of the context words that are tested along with the critical words. In Experiment 2, we used medium- and highfrequency words as context words, rather than the lowfrequency words used in Experiment 1, in order to increase the number of context words that were perceptually identified. A second difficulty in Experiment 1 was that the between-subject comparison of the effect of proportion overlap was made relatively insensitive by the presence of large individual differences in perceptual identification performance. To produce a more sensitive test, proportion overlap was varied within subjects in Experiment 2.
A low-overlap test was followed by a high-overlap test of perceptual identification. Another change between the experiments was that the presentation of a visual mask preceded, as well as followed, the presentation of a word for a test of its perceptual identification in Experiment 2, whereas a mask was presented only after test words in Experiment 1. The premask was meant to allow more precise control of the subjects' perceptual identification performance by making the test more difficult and, consequently, more sensitive to changes in the presentation duration of test words.

Unfortunately, the within-subject manipulation of proportion overlap unavoidably confounded test order with proportion overlap. The ordering of test conditions used was meant to produce lack of awareness followed by awareness of the relation between study and test. Presenting the conditions in the opposite order (high overlap followed by low overlap) would be to attempt to produce awareness followed by lack of awareness, a combination that might be impossible to achieve. However, one does need some means of protecting against problems for interpretation of results produced by any interactions of test order with the variables of interest. To do so, we combined a between-subject manipulation of type of test (recognition vs. perceptual identification) with the withinsubject manipulation of proportion overlap. One way that order effects could complicate interpretation of any interaction of study processing with proportion overlap in perceptual identification performance would be for recognition memory performance to be substantially poorer on the second test than on the first test. Comparisons of effects on the two types of test can be used to show that problems for interpretation of that sort did not arise.

\section{Method}

Subjects and Design. Forty-two students were paid to partici pate in Experiment 2: 24 in the perceptual identification task and 18 in the recognition memory task. The subjects in the recognition memory condition were tested after those in the perceptual identification condition. Originally, we did not include a recognition memory test condition in Experiment 2 because the results of Experiment 1 and the results of other experiments done in our laboratory convinced us that it was unnecessary to do so. Reviewers were unconvinced by our arguments, so we added the recognition memory test condition. The design was the same as in Experiment 1, with the exceptions that proportion overlap was varied within subjects and the type of test was varied between subjects. Four perceptual identification subjects were replaced because their level of performance on new items in the perceptual identification task was above $70 \%$ correct. Replacing the subjects did not alter the pattern of results.

Materials. A pool of 254 five-letter nouns that included 70 highfrequency words (greater than 50 occurrences per million), 70 medium-frequency words (20-29 occurrences per million), and 114 low-frequency words (1-5 occurrences per million), as scaled by Thorndike and Lorge (1944), served as materials. Twenty-four of those low-frequency words were presented in the test of perceptual identification given in Phase 1 to set a presentation duration to be used for the tests given in Phase 3 . Of the remaining 230 words, 120 words were presented in Phase 2 ( 30 high-, 30 medium-, and 60 low-frequency words). In that list, half of the words at each level of frequency in the language were presented in normal form 
to be read and half were presented as anagrams to be solved. Anagrams were constructed in the same way as in Experiment 1

Twenty of the low-frequency words that were read and 20 of the low-frequency words generated as a solution to an anagram, along with 20 new low-frequency words, served as critical words for the two tests of perceptual identification given in Phase 3. Each of those two tests contained 10 critical words of each of the three types. The two test lists differed in their proportion overlap with the list presented in Phase 2 . The low-overlap test list contained 120 words, 20 of which were old (10 old-read and 10 old-generated critical words); it began with 40 new words that included 20 high-and 20 medium-frequency words presented in random order. The remainder of the low-overlap test list was made up of 50 additional new words (20 high-, 20 medium-, and 10 low-frequency words) that were randomly intermixed with the 30 critical test words. The high-overlap test list contained 110 words of which 100 were old; it began with 30 old words that included 10 high-, 10 medium-, and 10 lowfrequency words presented in a random order. The remainder of the high-overlap test list was made up of 50 additional old words (20 high-, 20 medium-, and 10 low-frequency words) intermixed with the 30 critical words. Half of the old words in the high-overlap test list at each level of frequency in the language were earlier read and half were earlier given as a solution to an anagram. Six formats were generated by rotating the sets of 10 critical words through conditions (read, anagram, and new) and test lists (high- and lowoverlap). Each format was used equally often. The study and test lists were identical for the perceptual identification and recognition memory versions of the experiment.

Procedure. For the perceptual identification group, a test of perceptual identification was given in Phase 1 of the experiment to set the subject's level of performance on new words at a level of approximately $50 \%$ accuracy. The procedure for that test and the procedure for the two perceptual identification tests given in Phase 3 were generally the same as those in Experiment 1 . The only difference was that, following the $750-\mathrm{msec}$ presentation of the fixation bars and prior to the presentation of the test word, a 500-msec random-dot mask was presented. Thus, there was a 500-msec mask presented immediately preceding, as well as immediately following, the test word. In Phase 3, the low-overlap test was followed by the high-overlap test. There was a break between the two test sets during which the subject was told that the items in the second set would be made up almost entirely of words that were read or generated as a solution to an anagram in Phase 2 (no mention of any relation to the words in the anagram-solving phase was made prior to the low-overlap test).

For the recognition memory group, the initial perceptual identification test was not required; thus, the anagram-solving task was the first phase in the procedure. The list presented and the procedure for that task were the same as for the perceptual identification subjects and was immediately followed by the first (low overlap) recognition memory test. Words were presented one at a time (with presentation controlled by the subject) on the computer screen. The subject was instructed to indicate, by a keypress, whether the word on the screen was seen, either spelled correctly or in a scrambled form, in the previous anagram-solving phase of the experiment. The words were always spelled properly for the recognition test. The subject was informed that only a very few of the words were in fact old words, most would be new words. The second (high overlap) recognition memory test immediately followed the first, with a break only for instructions. The subject was informed that the recognition task remained the same but that now most of the words would be old with only a few new ones.

\section{Results and Discussion}

Recognition memory. The results of the recognition memory test are shown in Table 2 . A $2 \times 2$ within-subject ANOVA, with task at study (read vs. anagram) and degree
Table 2

Percent Correct Recognition Memory and

Perceptual Identification Performance in Experiment 2

\begin{tabular}{cccc}
\hline \multirow{2}{*}{$\begin{array}{c}\text { Overlap } \\
\text { Condition }\end{array}$} & \multicolumn{3}{c}{ Item Type } \\
\cline { 2 - 3 } New & Old-Read & Old-Generated \\
\hline \multicolumn{4}{c}{ Recognition Memory } \\
High & 90.6 & 47.2 & 77.8 \\
Low & 95.0 & 41.1 & 68.9 \\
& \multicolumn{5}{c}{} \\
High & 35.0 & 63.3 & 49.2 \\
Low & 38.8 & 50.4 & 45.8 \\
\hline
\end{tabular}

of overlap (high vs. low) as factors, showed a large recognition advantage for words that were generated from an anagram in the study phase over words that were read $\left[F(1,17)=29.2, M S_{e}=5.24\right]$. Neither the main effect of proportion overlap $\left[F(1,17)=1.34, M S_{\mathrm{e}}=7.54\right]$ nor the interaction of proportion overlap with study processing $(F<1)$ approached significance. A separate analysis that included the probability of false recognition for new words, as well as the probabilities of correct recognition for words earlier read or for words earlier generated, also failed to reveal a significant interaction with proportion overlap. However, recognition memory performance, as measured by the difference between hits and false alarms, was numerically larger in the high-overlap test condition than in the low-overlap test condition. This was true although the test order was such that the delay between study and test was longer for the high-overlap test. Removing the effect of test order might produce a significant effect of proportion overlap, allowing one to show that reinstating study context enhances recognition memory performance.

Perceptual identification. The presentation duration employed for perceptual identification ranged from 33 to $67 \mathrm{msec}$ and had a mean of $49.5 \mathrm{msec}$. The probability of correctly identifying words is shown in Table 2 (separately for each combination of conditions). First, we compared the perceptual identification of words that were earlier read with that of new words. The results of that analysis showed a large advantage in identification performance for old words (56.9\%) over new words (36.9\%) $\left[F(1,23)=28.49, M S_{\mathrm{e}}=337.0\right]$. More importantly, the interaction between proportion overlap and prior presentation was highly significant $\left[F(1,23)=12.43, M S_{\mathrm{e}}=\right.$ 134.1]. Replicating results reported by Jacoby (1983a), the advantage in identification performance of words that had been read earlier, as compared with new words, was larger when the majority of the tested words were old rather than new.

The finding of a large effect of proportion overlap was important to allow us to choose between different accounts of that effect. Again, an intentional memory interpretation of the proportion overlap effect would predict a larger effect of increasing proportion overlap for words earlier generated as a solution to an anagram than for words that were earlier read. An analysis that included data from only 
the two types of old words showed that words that were previously read $(56.9 \%)$ were more likely to be identified than were words that were earlier generated $(47.5 \%)$ $\left[F(1,23)=9.97, M S_{\mathrm{e}}=211.5\right]$. The main effect of proportion overlap was also significant $[F(1,23)=5.07$, $M S_{\mathrm{e}}=312.6$ ]. Old test words of both types were more likely to be identified when the majority of the test words were old $(56.3 \%)$ than when they were new $(48.1 \%)$. Most importantly for our purposes, the interaction between proportion overlap and earlier reading versus generating a word approached significance $[F(1,23)=3.15$, $\left.M S_{\mathrm{e}}=175.0, p<.10\right]$. The form of that interaction is the opposite of the form that would be predicted by an intentional memory interpretation of the proportion overlap effect. Although the subjects were less able to recognize words that were read as compared with those that were generated, words that were earlier read benefited marginally more from increasing proportion overlap.

The results of Experiment 2 make it safe to conclude that intentional use of memory for earlier presented words is not necessary to produce the effect of increasing proportion overlap between study and test on perceptual identification performance. A comparison of effects in the perceptual identification and recognition memory test conditions discourages any attempt to save an intentional memory account by appealing to the confounding of test order with the manipulation of proportion overlap. The effects of proportion overlap on recognition memory performance were not significant and, numerically, the form of the interaction between study processing and proportion overlap was the opposite for the two test conditions. The recognition advantage of words presented as anagrams over those that were read was numerically larger on the high-overlap test than on the low-overlap test and, therefore, interpretation of the interaction of study processing with proportion overlap in perceptual identification performance is not compromised by an effect of test order on recognition memory performance. A comparison of the results of the two experiments shows that the changes in procedure did produce an effect of proportion overlap in Experiment 2 that was highly significant when tested statistically, whereas the effect in Experiment 1 was only of borderline significance. This difference was largely due to a reduction in error variance produced by our use of a within-subject design in Experiment 2.

To dismiss any simple intentional memory account of the proportion overlap effect on perceptual identification performance, it is only necessary to show that increasing proportion overlap does not have a larger effect for words that are easy than for those that are difficult to recognize or recall as previously presented. This essentially affirms the null hypothesis. However, results of Experiment 2 went beyond that requirement by showing a marginally significant interaction of a form opposite to that predicted by an intentional memory account. By adding subjects to our experiment or by using an even stronger manipulation of test context, it might be possible to produce a significant interaction of that sort in perceptual identification performance and, perhaps, even a significant inter- action of an opposite form for recognition memory performance. If so, the interactions could be interpreted as showing that the effect of increasing accessibility by reinstating study context depends on the compatibility of the prior processing of a word and the processing demanded by the test of retention. Nevertheless, at present, we are satisfied to conclude that effects of increasing proportion overlap on perceptual identification performance are not mediated by an intentional use of memory. This is not to say that intentional uses of memory never contribute to proportion overlap effects but, rather, that an intentional use of memory is not necessary to produce a proportion overlap effect.

By the episodic view, the effects of prior experience depend on the similarity between the study and the test processing of a word. The advantage in identification performance of words that were earlier read over those that were earlier generated is in line with the claimed importance of processing similarity. The processing required to read a word is more similar to that required to identify the word (essentially, a test of reading) than is the processing required to generate a word. Reinstating study context makes it more likely that memory for earlier processing of a word will be retrieved and will enhance perception when the word is later presented for a test of its perceptual identification.

\section{GENERAL DISCUSSION}

In sum, words that were earlier read were more likely to be identified later but less likely to be recognized as old than were words that were earlier generated. Increasing the proportion overlap between study and test increased the effect of a prior presentation on perceptual identification performance. The effect of increasing proportion overlap did not rely on the intentional use of memory for earlier presented words: Contrary to results that would be expected if the effects of proportion overlap were produced by an aware use of memory, words that were likely to be recognized as old (words that were generated) did not benefit more from increasing proportion overlap than did words that were unlikely to be recognized as old (words that were read). Awareness that a word has been previously presented is not necessary for effects of memory for prior episodes on word identification nor is such awareness necessary for the effectiveness of manipulations of test context. More generally, effects of reinstating study context on an indirect test of memory do not necessarily rely on an intentional use of memory. The finding of an effect of reinstating study context on unconscious influences of memory was predicted by an episodic view, but it would not be predicted by views holding that performance on an indirect test relies on a different memory system than does performance on a direct test of memory (e.g., Cohen \& Squire, 1980; Tulving, 1983).

The results of both experiments showed that increasing proportion overlap increased the probability of identification for old words but produced a slight decrease in 
the probability of identification for new words. A similar pattern of results was observed by Jacoby (1983a) and was interpreted as showing that, in terms of signal detection theory, manipulating proportion overlap influences criterion or response bias rather than sensitivity: The increased accessibility of memory for prior episodes that comes from increasing proportion overlap influences the interpretation of perceptual data in a way that biases toward the perception of old words and interferes with that of new words. Ratcliff, McKoon, and Verwoerd (1989) have also presented evidence to show that effects of prior experience on identification performance are produced by a change in criterion rather than by a change in sensitivity. It is important to note that attributing effects to a change in criterion is not to deny that the effects are truly perceptual. The effects of prior experience on identification are perceptual in that they do not arise from conscious guessing of old words (Jacoby, Allan, Collins, \& Larwill, 1988). It is likely that, dependent on the details of the situation, memory for prior episodes can produce either criterion or sensitivity effects on performance of perceptual tasks.

Effects of memory for prior episodes on word perception are problematic for those who claim that word perception relies on a module, such as a logogen system, that is separate from the rest of memory (e.g., Morton, 1979). The priming of a logogen would produce effects on word identification that are very general, effects that are influenced neither by manipulations of study processing nor by test context. A modular system will thus have trouble explaining the effects of reading versus generating a word. To preserve a modularity view, some (e.g., Forster \& Davis, 1984) have explained effects of memory for prior episodes on word identification as reflecting an aware use of memory; however, results of the present experiments rule against that interpretation. To accommodate our results, it could be claimed that word perception relies on unaware use of memory for prior episodes, as well as a separate module. However, doing so weakens claims of modularity and leaves one with the task of sorting out effects of the two types.

Unconscious influences of context are common in everyday circumstances and, if unaccompanied by conscious recollection, may lead to confusion. An example from the second author's experience involves a case of something akin to unconscious plagiarism. A graduate teaching assistant, following a lecture given by the author in an introductory class, suggested an anecdote that would have illustrated one of the topics of the day's class very well. The anecdote was in fact one that the author had used the previous year in precisely that situation, with the assistant present. The assistant, however, claimed to have heard the anecdote not from the author but from his own undergraduate instructor. This appears to be a case of "unconscious plagiarism," because the assistant's undergraduate instructor denied ever having heard the anecdote (which the author had actually invented). The point is that the story came to mind for the assistant in a con- text very similar to that in which it was earlier heard, although he obviously did not recollect hearing the story in that context. Unconscious context effects are normally benign and obviously quite useful in aiding memory appropriate to the situation. They can, however, leave us in the awkward position where, if we fail to consciously retrieve the source, we are most likely to repeat a story to the very person from whom we first heard it. Jacoby, Kelley, and Dywan (1989) review evidence of effects of this sort.

Retrieval of memory for prior episodes allows performance to be quickly adapted to local circumstances and is important for performance on categorization, as well as perceptual, tasks. Allen and Brooks (in press) have recently extended the manipulation of proportion overlap to a categorization task. They found that when the majority of the instances of a category presented at test were the same as presented during study, people were more likely to categorize new items on the basis of analogy to old items. This was true even though categorization on the basis of analogy was in conflict with a simple rule that subjects had been told to use for categorizing stimuli. Perception and categorization most often occur in familiar environments, so that memory for what was previously done in that environment serves as a good guide for current processing. Memory for prior episodes can serve to rapidly adapt processing to an environment.

The importance of test context and other retrieval factors have been relatively neglected in both studies of perception and those of concept learning. One reason for this neglect is that retrieval of memory for prior episodes has traditionally been thought of as necessarily involving awareness of memory for the prior episodes as assessed by tests of recognition memory or recall. Recognition memory or recall of a prior encounter with an item seems too slow and error-prone to be of assistance for perception and categorization. However, gaining awareness that one has had a prior experience involves different activities than does using memory for that prior experience to aid performance of a current task (e.g., Jacoby \& Kelley, 1987; Jacoby et al., 1989). Our studies show that effects of memory for prior episodes need not rely on the ability to recall or recognize previously presented items. It may be gaining awareness of memory for prior episodes, rather than the use of those memories as a guide for later processing, that is slow and prone to error.

\section{REFERENCES}

ALLEN, S. W., \& BRooks, L. R. (in press). Specializing the operation of an explicit rule. Joumal of Experimental Psychology: General. Cohen, N. J., \& Squire, L. R. (1980). Preserved learning and retention of pattern-analyzing skill in amnesia: Dissociation of knowing how and knowing that. Science, 210, 207-210.

ForSTER, K. I., \& DAVIS, C. (1984). Repetition priming and frequency attenuation in lexical access. Joumal of Experimental Psychology. Learning, Memory, \& Cognition, 10, 680-698.

Graf, P., \& Schacter, D. L. (1985). Implicit and explicit memory for new associations in normal and amnesic subjects. Journal of Experimental Psychology: Learning, Memory, \& Cognition, 11, 501-518. 
Holender, D. (1986). Semantic activation without conscious identification in dichotic listening, parafoveal vision, and visual masking: A survey and appraisal. Behavioral \& Brain Sciences, 9, 1-23.

JACOBY, L. L. (1978). On interpreting the effects of repetition: Solving a problem versus remembering a solution. Journal of Verbal Learning \& Verbal Behavior, 17, 649-667.

JACOBY, L. L. (1983a). Perceptual enhancement: Persistent effects of an experience. Journal of Experimental Psychology: Learning, Memory, \& Cognition, 9, 21-38.

JACOBY, L. L. (1983b). Remembering the data: Analyzing interactive processes in reading. Journal of Verbal Learning \& Verbal Behavior, 22, 485-508.

Jacoby, L. L., Allan, L. G., Collins, J. C., \& Larwill, L. K. (1988). Memory influences subjective experience: Noise judgments. Journal of Experimental Psychology: Learning, Memory, \& Cognition, 14, 240-247.

JACOBY, L. L., \& BRooKs, L. R. (1984). Nonanalytic cognition: Memory, perception and concept learning. In G. H. Bower (Ed.), The psychology of leaming and motivation: Advances in research and theory (Vol. 18, pp. 1-47). New York: Academic Press.

JACOBY, L. L., \& DALLAS, M. (1981). On the relationship between autobiographical memory and perceptual learning. Journal of Experimental Psychology: General, 3, 306-340.

JACOBY, L. L., KELLEY, C. M. (1987). Unconscious influences of memory for a prior event. Personality \& Social Psychology Bulletin, 13, 314-336.

JaCOBY, L. L., Kelley, C. M., \& DywaN, J. (1989). Memory attributions. In H. L. Roediger \& F. I. M. Craik (Eds.), Varieties of memory and consciousness: Essays in honour of Endel Tulving (pp. 391-422). Hillsdale, NJ: Erlbaum.

JOHNSON, M. K., \& HASHER, L. (1987). Human learning and memory. Annual Review of Psychology, 38, 631-668.

MoRTON, J. (1979). Facilitation in word recognition: Experiments causing change in the logogen model. In P. A. Kolers, M. E. Wrolstal, \& H. Bonma (Eds.), Processing of visible language (Vol. 1, pp. 259268). New York: Plenum.

Moscovitch, M., Winocur, G., \& McLachlan, D. (1986). Memory as assessed by recognition and reading time in normal and memoryimpaired people with Alzheimer's disease and other neurological disorders. Joumal of Experimental Psychology: General, 115, 331-347.
Ratcliff, R., McKoon, G., \& Verwoerd, M. (1989). A bias interpretation of facilitation in perceptual identification. Journal of Experimental Psychology: Learning, Memory, \& Cognition, 15, 378-387.

RichaRDSON-KLAVEHN, A., \& BJoRK, R. A. (1988). Measures of memory. Annual Review of Psychology, 39, 475-543.

RoEdiger, H. L., \& BlaXton, T. A. (1987). Retrieval modes produce dissociations in memory for surface information. In D. S. Gorfein \& R. R. Hoffman (Eds.), Memory and cognitive processes: The Ebbinghaus centennial conference. Hillsdale, NJ: Erlbaum.

SCHACTER, D. L. (1985). Multiple forms of memory in humans and animals. In N. M. Weinberger, J. L. McGaugh, \& G. Lynch (Eds.), Memory systems of the brain (pp. 351-379). New York: Guilford.

Schacter, D. L., \& Graf, P. (1986). Effects of elaborative processing on implicit and explicit memory for new associations. Journal of Experimental Psychology: Learning, Memory, \& Cognition, 12, 432-444.

Slamecka, N. J., \& Graf, P. (1978). The generation effect: Delineation of a phenomenon. Jourmal of Experimental Psychology: Human Learning, \& Memory, 4, 592-604.

THORNDIKE, E. L., \& LORGE, I. (1944). The teachers' handbook of 30,000 words. New York: Columbia University Press.

Tulving, E. (1983). Elements of episodic memory. London: Oxford University Press.

Whittlesea, B. W. A. (1987). Preservation of specific experiences in the representation of general knowledge. Journal of Experimental Psychology: Learning, Memory, \& Cognition, 13, 3-17.

\section{NOTES}

1. If a comparable analysis is carried out on the critical items for which perceptual identification scores were obtained, the scores are higher overall and the same effect, albeit weakened, is found. In the low-overlap condition, generated items were recognized better $(87 \%)$ than were read items $(77 \%)[t(23)=2.71]$. In the high-overlap condition, again the generated items were recognized better $(90 \%)$ than were read items $(78 \%)$ $[t(22)=3.32]$.

(Manuscript received February 17, 1989; revision accepted for publication September 18, 1989.) 